\title{
A rare case of hemangioma arising from the azygos vein: Informative procedure with endobronchial ultrasonography
}

\author{
Sumitaka Yamanaka, MD, Akira Sakurada, MD, Yuji Matsumura, MD, Chiaki Endo, MD, Masami Sato, MD, and \\ Takashi Kondo, MD, Sendai, Japan
}

\section{Clinical Summary}

A 52-year-old woman with a previous history of vasospastic angina presented with an asymptomatic mediastinal mass on her routine follow-up chest roentgenogram. Values shown by means of hematologic and biochemical examinations were within normal range. A computed tomographic scan demonstrated a mass of 40 $\mathrm{mm}$ in diameter with a smooth margin adjacent to the trachea, superior vena cava, and ascending aorta. It showed high enhancement, although the degree of enhancement was lower than that of the surrounding vascular structures. Because a vascular tumor was suspected, digital subtraction angiography was performed, but this did not show feeder arteries or other vascular staining. Magnetic resonance imaging suggested high intensity on the T1-weighted image and low intensity on the T2-weighted image. No flow void was demonstrated (Figure 1, A). A diagnosis of lymphoadenopathy, such as Castleman disease or a malignancy, was given by radiologists. An ordinary bronchoscopic examination also showed no abnormal lesion. XBF-UC40P, a new prototype bronchofiberscope with an electrical curved linear array method, clearly showed a cystic mass with blood flow by using the power Doppler mode (Figure 1, $B$ ). No definite findings were detected on abdominal and cardiac echocardiographs.

Because a vascular tumor was strongly suggested on the basis of endobronchial ultrasonography (EBUS), surgical removal of the mass by means of video-assisted thoracotomy was performed. Macroscopically, the mass seemed to be a varix of the azygos vein. No abnormal findings were observed in the superior vena cava and right atrium. The wall of the cyst was very thin, and delicate manipulation was absolutely necessary in dissecting the tumor (Figure 2, A). Pathologic examination of the excised tumor showed it to be a hemangioma composed of various cavities separated by fine septa lined with attenuated endothelium not unlike the hemangiomas of other regions. The wall of the tumor was composed of

\footnotetext{
From the Department of Thoracic Surgery, Institute of Development, Aging and Cancer, Tohoku University, Sendai, Japan.

Received for publication Aug 11, 2003; accepted for publication Aug 25, 2003.

Address for reprints: Masami Sato, MD, Department of Thoracic Surgery, Institute of Development, Aging and Cancer, Tohoku University, Sendai, Japan (E-mail: m-sato@idac.tohoku.ac.jp).

J Thorac Cardiovasc Surg 2004;127:294-5

$0022-5223 / \$ 30.00$

Copyright $\odot 2004$ by The American Association for Thoracic Surgery

doi:10.1016/j.tcvs.2003.08.025
}

smooth muscle and connective tissues (Figure 2, B). The postoperative course was uneventful.

\section{Discussion}

Although many kinds of tumors originate from the mediastinal structures, vascular tumors of the azygos vein are extremely rare. In fact, to the best of our knowledge, there has been only one case report of such a tumor ${ }^{1}$ and no reports of hemangioma of the azygos vein. It is very difficult to establish a preoperative diagnosis for this kind of tumor only by means of radiologic examination. On the basis of the findings of digital subtraction angiography, which did not show definite feeder arteries or vascular staining, our radiologists considered the tumor to be a solid tumor, such as a lymphoadenoma. The azygos vein is hardly enhanced in angiography, even by means of phlebography. Also, its slow turbulent flow probably prevents the appearance of a flow void on chest magnetic resonance. ${ }^{2}$

A new prototype, EBUS, however, showed information crucial for understanding the details of the mass. EBUS revealed that the mass contained cystic structures with blood flow, which was detected by using the power Doppler mode. This finding was sufficient for us to avoid the performance of transtracheal needle aspiration biopsy-cytology or percutaneous needle aspiration, which might have led to bleeding. In cases of right paratracheal cystic tumor, we must also be careful because the mass could be a varix of the azygos vein ${ }^{3-5}$ or a vascular tumor. EBUS with a power Doppler device provides crucial information for the diagnosis of vascular tumors, especially in this zygous vein area. Although this is the very first EBUS with the power Doppler mode, we are convinced that this procedure is very useful in accurately diagnosing masses in the paratracheal area.

We appreciate the assistance of Olympus Optical, Co, Ltd, in the use of the prototype bronchofiberscope XBF-UC40P.

\section{References}

1. Nataf P, Regnard JF, Solvignon F, et al. Epitheloid hemangioendothelioma of the azygos vein. Arch Mal Coeur Vaiss. 1989;82:1919-22.

2. Kurihara Y, Nakajima Y, Ishikawa T. Saccular aneurysm of the azygos vein simulating a paratracheal tumor. Clin Radiol. 1993;48:427-8.

3. Francis JP, Albert DS, Ari OH, et al. Giant azygos vein varix. Ann Thorac Surg. 1997;63:1167-9.

4. Seebauer L, Prauer HW, Gmeinwieser J, et al. A mediastinal tumor simulated by a sacculated aneurysm of the azygos vein. Thorac Cardiovasc Surg. 1989;37:112-4.

5. Olbert F, Kobinia G, Russe OJ, et al. Aneurysmal dilatation of the bulb of the azygos vein. Wien Med Wochenschr. 1980;65:1-26. 

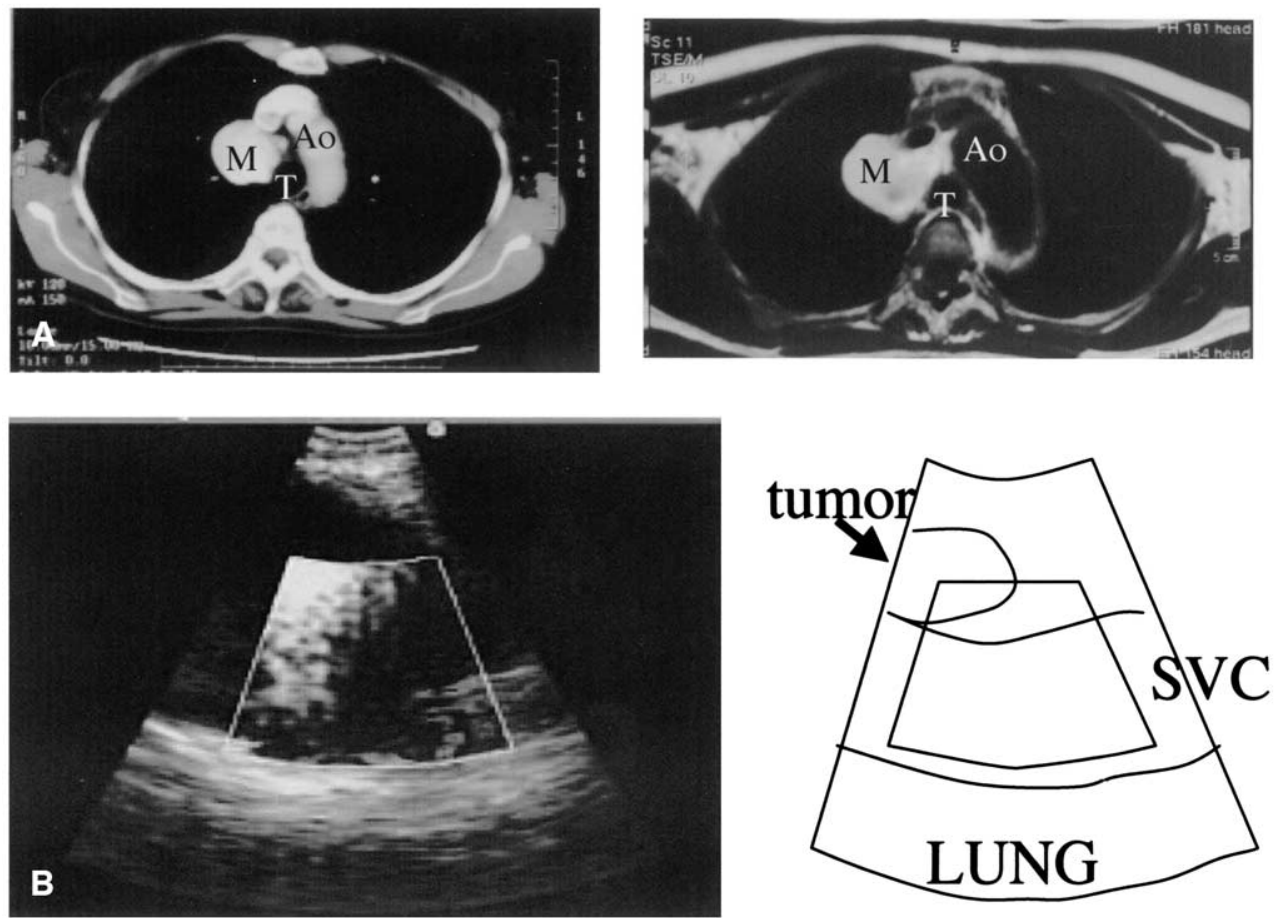

Figure 1. A, A chest computed tomographic scan demonstrated a mass $(M)$ with high enhancement adjacent to the right wall of the trachea $(T)$. Magnetic resonance imaging showed a mass with high intensity on the T1-weighted image. No flow void was observed. Ao, Aorta. Left, Chest computed tomographic image with mediastinal window. Right, T1-weighted image with magnetic resonance imaging of the chest. B, EBUS revealed the mass to be cystic, with high blood flow by the power Doppler mode. The area surrounded by the white line indicates the area subjected to power Doppler analysis.
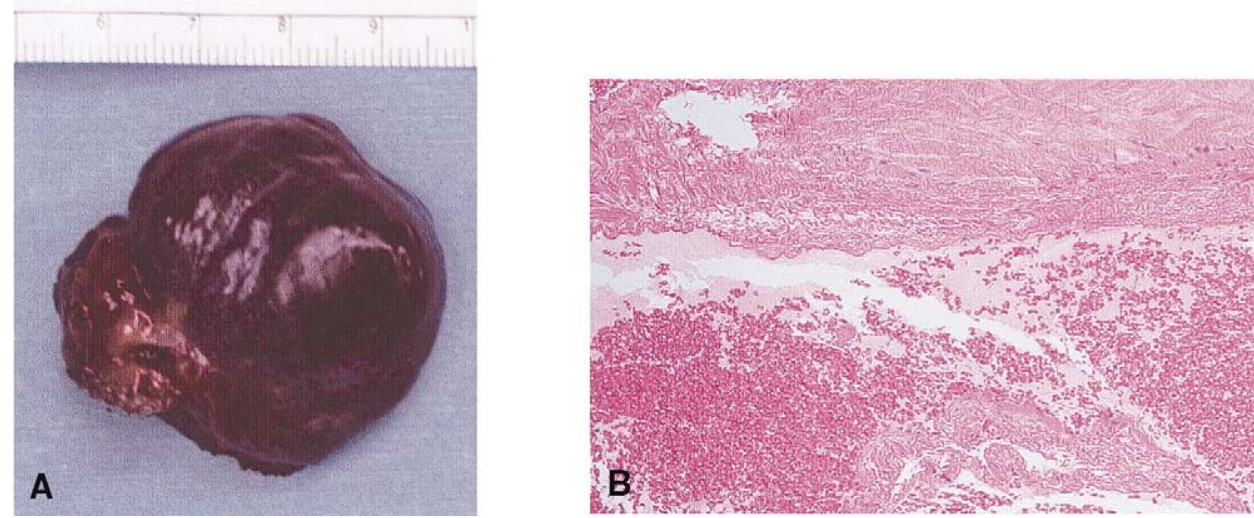

Figure 2. A, Macroscopic findings of the resected specimen. B, Microscopic findings showing various cavities filled with red cells separated by fine septa lined with attenuated endothelium are compatible with the diagnosis of hemangioma. 